\title{
How to assess and predict success or failure of intra-detrusor injections with onabotulinumtoxinA
}

\author{
Mikolaj Przydacz ${ }^{A-F}$, Tomasz Golabek ${ }^{A, E, F}$, Piotr Chlosta ${ }^{A, E, F}$ \\ Department of Urology, Jagiellonian University Medical College, Kraków, Poland \\ A - research concept and design; B - collection and/or assembly of data; $\mathrm{C}$ - data analysis and interpretation; \\ $D$ - writing the article; $E$ - critical revision of the article; $F$ - final approval of the article
}

Address for correspondence

Mikolaj Przydacz

E-mail: mikolaj.przydacz@yahoo.com

Funding sources

None declared

Conflict of interest

None declared

Received on February 5, 2018

Reviewed on May 2, 2018

Accepted on May 5, 2018

Published online on February 5, 2019

\begin{abstract}
Intra-detrusor injection therapy with onabotulinumtoxinA is generally accepted as a highly effective, minimally invasive and well-tolerated day procedure for patients with refractory overactive bladder (OAB) and neurogenic detrusor overactivity (NDO). The aim of this study was to summarize currently available methods of assessing treatment efficacy and risk factors that may influence the therapeutic effect of this approach. We found that there are discrepancies in the assessment methods. The evaluation of intra-detrusor injections with onabotulinumtoxinA in clinical trials are not always transposable into day-to-day practice. Moreover, the primary endpoints in clinical trials do not explore the entirety of meaningful patient-centered outcomes. Therefore, in daily clinical practice with patients with overactive bladder syndrome, the therapy should be assessed with objective measures (bladder diaries) and patient-oriented outcomes analyzing the quality of life (questionnaires). In neurogenic individuals, therapeutic efficacy should be additionally evaluated with urodynamic studies. Potential risk factors that may influence the treatment outcomes include urodynamically proven detrusor overactivity, elevated maximum detrusor pressure, greater maximum cystometric capacity, impaired bladder compliance, older age, male gender, a higher baseline bother score, previous anticholinergic treatment, and repeated injections with a subsequent decline in efficacy. The risk of intermittent catheterization following injections seems to depend on the etiology of detrusor overactivity, the injected dose, the injection technique, and the definition of significant post-void residual urine requiring catheterization.
\end{abstract}

Key words: onabotulinumtoxinA, failure, risk factors
Cite as

Przydacz M, Golabek T, Chlosta P. How to assess and predict success or failure of intra-detrusor injections with onabotulinumtoxinA. Adv Clin Exp Med. 2019;28(4):555-567. doi:10.17219/acem/90764

DOI

10.17219/acem/90764

Copyright

Copyright by Author(s)

This is an article distributed under the terms of the

Creative Commons Attribution Non-Commercial License

(http://creativecommons.org/licenses/by-nc-nd/4.0/) 
Intra-detrusor injection therapy with onabotulinumtoxinA (Botox ${ }^{\circledR}$; Allergan Inc., Irvine, USA) is generally accepted as a highly effective, minimally invasive and well-tolerated day procedure for patients suffering from refractory overactive bladder $(\mathrm{OAB})$ and neurogenic detrusor overactivity (NDO). Its efficacy has been widely investigated and proven in systematic reviews with metaanalyses of randomized, double-blind, placebo-controlled multicenter trials, ${ }^{1-3}$ and onabotulinumtoxinA is currently recommended by the leading urological societies for both neurogenic and non-neurogenic voiding dysfunctions. ${ }^{4}$ Treatment with onabotulinumtoxin A in patients suffering from OAB mainly improves their quality of life and reduces incontinence, while in those with NDO it additionally helps preserve renal function.

OnabotulinumtoxinA, a specific formulation of botulinum toxin, is a neuromodulator that directly inhibits efferent acetylcholine-mediated detrusor contractions and reduces muscle spasticity. ${ }^{3}$ This therapy also has other mechanisms of action, and inhibits the release of different vesicle-mediated neurotransmitters responsible for inappropriate afferent signaling from an overactive detrusor, including effects on afferent sensory neurons via TRPV1 and P2X3 receptors. Therefore, the ability of onabotulinumtoxin A to synergistically target the afferent and efferent neuronal pathways of bladder control may explain the profound effect observed on urgency, frequency and urgency incontinence, as well as the failure of prior anticholinergic therapy. Consequently, onabotulinumtoxin A offers a complex inhibitory effect on multiple targets in the bladder wall that may cause OAB and NDO.

It is of utmost importance to identify patients in whom intra-detrusor Botox ${ }^{\circledR}$ therapy has failed to help or who may not benefit from it. However, appropriate patient enrollment and assessment of treatment success/failure may be challenging for physicians, as these are generally based on subjective outcomes. There is no single study summarizing currently available assessment methods of therapy success/failure and predicting factors for this treatment. Recognition of predictors is a prerequisite for an individualized approach, and adequate knowledge could help clinicians avoid ineffective procedures and potential treatmentrelated adverse effects. Furthermore, assessment methods of response/non-response to the treatment have important applications in future research. Therefore, the aim of this review is to summarize methods of assessing the success/ failure of the therapy and to present a list of risk factors that may influence the outcomes of intra-detrusor injections of onabotulinumtoxinA.

\section{Material and methods}

Maximum data were collected according to different methods, including searches with multiple and specific keywords and reference checks. Our searches targeted the terms "neurogenic bladder", "neurogenic lower urinary tract dysfunction", "overactive bladder", "onabotulinumtoxin A", "success", "failure", and "risk factors". We searched PubMed (including MEDLINE and PubMed Central), the Cochrane Library and the Web of Science databases. During full-text screening, references in pertinent papers were checked manually to identify other related papers. In order to present reliable assessment methods of treatment success/failure, only randomized double-blind placebo-controlled trials were included. They were also selected from currently available systematic reviews with meta-analyses of OAB and NDO to avoid omitting important trials. ${ }^{1-3,5-11}$ The reports on risk factors that we found (searching through all the available literature) were analyzed with a modified version of the Oxford grading system for recommendations, using levels of evidence (LE) and grades of recommendation (GR). ${ }^{12}$

\section{Results}

\section{Assessment methods}

There is no consensus among researchers how to assess the success/failure of treatment with onabotulinumtoxin $\mathrm{A}$ injections. To make matters even more complex, most of the randomized clinical trials on OAB and NDO used either objective measures from bladder diaries, subjective patient-reported outcomes from questionnaires or urodynamic-related parameters as primary experimental outcomes.

\section{Overactive bladder}

Overactive bladder can be debilitating for patients, and may have a profound negative impact on the patient's quality of life. Oral anticholinergics are the mainstay of first-line pharmacologic treatment, ${ }^{4}$ but may be associated with unwanted side effects including dry mouth, constipation and blurred vision, leading to poor compliance and a high discontinuation rate in clinical practice. Therefore, minimally invasive treatment with onabotulinumtoxin A offers an effective alternative to other minimally invasive therapies such as neuromodulation, as well as to invasive surgical bladder augmentation. Our study revealed that there is no homogeneity among randomized clinical trials in reporting outcomes. Detailed comparisons between published randomized placebo-controlled trials are hampered by differences in the outcome measures used, whether diary data were compared daily or weekly, and the fact that studies recruited both men and women. Clinicians should also keep in mind that our current knowledge regarding the efficacy of the treatment is limited to patients with $\mathrm{OAB}$ wet (i.e., OAB with concomitant urinary incontinence). There is a paucity of reliable data analyzing treatment 
with onabotulinumtoxin $A$ in individuals suffering from $\mathrm{OAB}$ without urinary incontinence (OAB dry), who comprise approx. $2 / 3$ of all OAB patients. ${ }^{13}$

To date, 9 randomized placebo-controlled clinical trials have been conducted in order to evaluate the efficacy of onabotulinumtoxin A in OAB. ${ }^{14-25}$ A summary of the patients included, the doses evaluated and primary and secondary experimental outcomes is presented in Table 1. The most common primary experimental outcome, used in 4 studies, has been defined as a change from baseline in the number of urgency urinary incontinence episodes. Three of these studies used an additional co-primary outcome, defined as the proportion of patients with a positive treatment response on the 4-score Treatment Benefit Scale (2 studies) and symptom scores assessed with a validated questionnaire (1 study). One study used a change from baseline in urinary frequency per $24 \mathrm{~h}$ as a primary experimental outcome, 1 study evaluated time to treatment failure (defined as a score of 4 or greater on the Patient Global Impression of Improvement) and 1 used the proportion of patients showing $>50 \%$ improvement compared to baseline of both urgency and urgency urinary incontinence episodes. Two studies mainly based their analyses on urodynamic parameters and used maximum cystometric capacity as a primary experimental outcome.

A time analysis of the studies included showed that the effects of onabotulinumtoxinA are commonly perceived between 3 days and 2 weeks after injection. ${ }^{14,17,22,24}$ Although the mean duration of onabotulinumtoxinA-related improvement is approx. 9-10 months, ${ }^{14,15}$ the efficacy of the therapy should be assessed between weeks 4 and 12, as some experimental outcomes have been shown to become insignificant, with decreasing treatment response compared with a placebo after 12 weeks (symptom reduction benefits typically show a slight trend towards decrease after 20-24 weeks). ${ }^{14,17,18,23,24}$ In patients with more severe OAB at baseline (i.e., more than 8 episodes of incontinence per day), efficacy should be assessed no sooner than 6 weeks after injection. ${ }^{16}$

\section{Neurogenic detrusor overactivity}

Neurogenic detrusor overactivity is one of the most challenging problems in urology. Various disorders or injuries affecting the central nervous system (e.g., stroke, Parkinson disease and multiple sclerosis) may cause this chronic dysfunction, in which the bladder becomes overactive and empties too frequently/quickly. The primary goals of treatment for NDO are to protect the upper urinary tract by decreasing bladder pressure, reducing incontinence and improving quality of life. Initial NDO treatment consists of anticholinergics, often with clean intermittent catheterization (CIC). However, long-term treatment may be suboptimal, with patients stopping medication due to a lack of efficacy and bothersome side effects, similar to those with OAB. Minimally invasive treatment with
onabotulinumtoxinA has been of benefit to those patients, as it induces paralysis of the detrusor muscle and consequently results in a decreased risk of vesicoureteral reflux, preventing upper urinary tract deterioration and possible kidney damage.

To date, 6 randomized placebo-controlled clinical trials have evaluated the efficacy of intra-detrusor injections of onabotulinumtoxinA in neurogenic patients. ${ }^{26-34}$ These studies mainly included patients with spinal cord injuries or with multiple sclerosis. All of these studies used a change from baseline in the number of urgency urinary incontinence episodes per week as the primary experimental outcome. Five of the studies assessed this primary experimental outcome at week 6 .

Improvements usually occur starting from week 2 and are maintained for the duration of 24 weeks of followup. ${ }^{27,30-34}$ In 3 studies with an open-label phase and longer follow-up, the median duration of the effect - the time lapse before patient-requested retreatment - was 36, 42.1 and 60 weeks, respectively. ${ }^{28,29,31}$ These benefits reached their maximum between the $2^{\text {nd }}$ and $6^{\text {th }}$ week. ${ }^{10}$ Approximately $60 \%$ of the patients may maintain the treatment effect for up to 9 months. ${ }^{34}$ However, some of the efficacy parameters tend to become insignificant after 18-24 weeks ${ }^{26,28}$ and efficacy evaluation before 6 weeks may be too early for reliable assessment. ${ }^{34}$

\section{Risk factors for treatment failure}

\section{Detrusor overactivity}

Detrusor overactivity has been defined as the presence of involuntary detrusor contractions during the filling phase of urodynamics. Whereas in neurogenic individuals urodynamically proven detrusor overactivity is necessary to establish a diagnosis of NDO, in those with OAB, although common, it is not present in all patients and not required for an OAB diagnosis.

The impact of detrusor overactivity on the final effect of treatment with onabotulinumtoxin $\mathrm{A}$ in $\mathrm{OAB}$ patients is still under debate, as the majority of available studies required detrusor overactivity for inclusion. Dowson et al. conducted a randomized controlled trial examining the effects of onabotulinumtoxinA exclusively in patients with $\mathrm{OAB}$ without concomitant detrusor overactivity (LE 1, GR B). ${ }^{21}$ The trial was halted after the recruitment of 23 patients as a result of poorly perceived patient benefit and no clinical benefit, with no change observed in the symptom score and quality of life for the majority of the participants. Individually, only 2 patients in the treatment arm of the study derived any benefit from the injections, whereas the remainder experienced no change in their symptoms. Of note, the study enrolled only $1 / 3$ of the patients suggested by the power calculation, and the primary endpoint (maximum cystometric capacity) was statistically significant. In a large randomized placebo-controlled clinical trial 


\begin{tabular}{|c|c|c|c|c|c|c|c|}
\hline 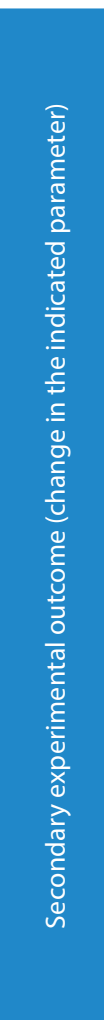 & 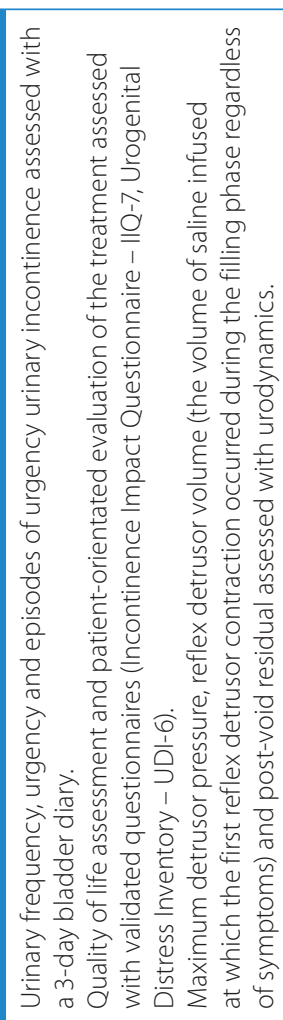 & 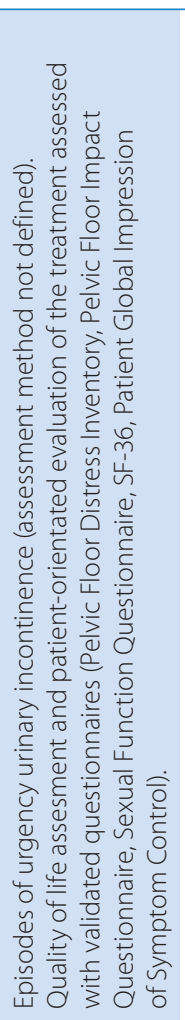 & 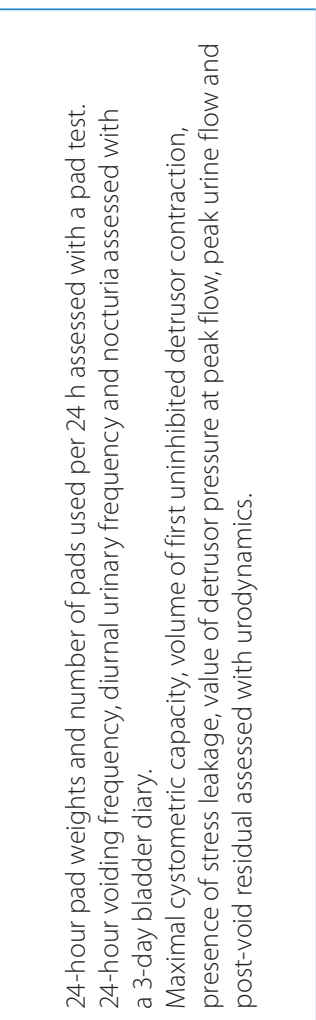 & 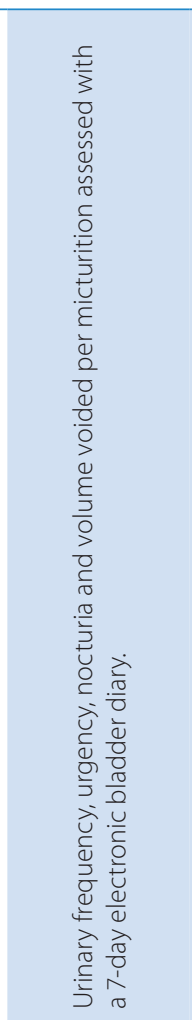 & 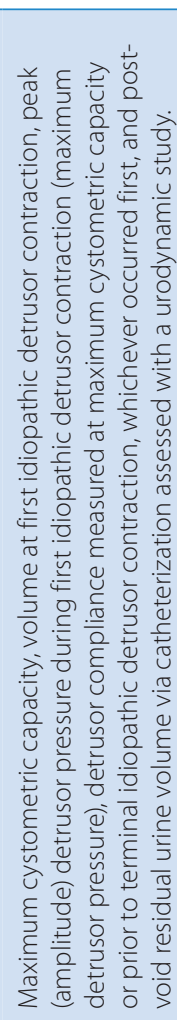 & 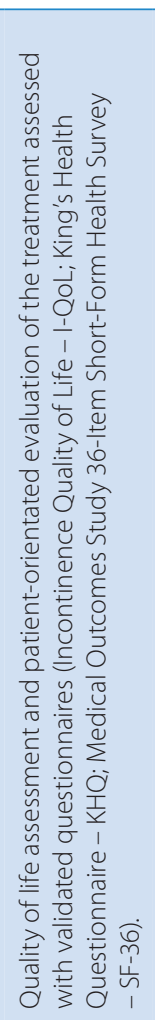 & 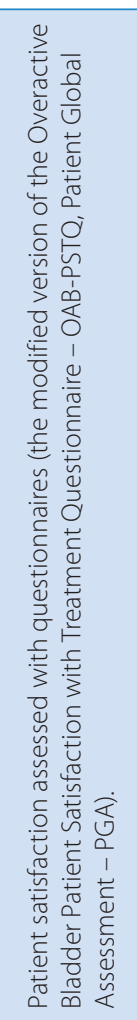 \\
\hline 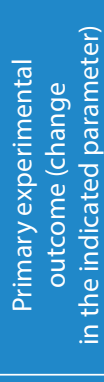 & 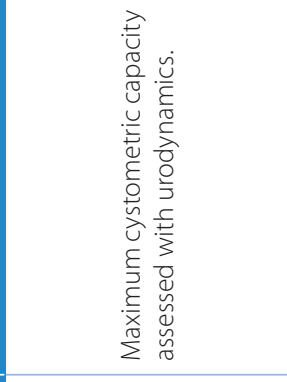 & 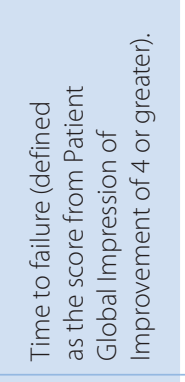 & 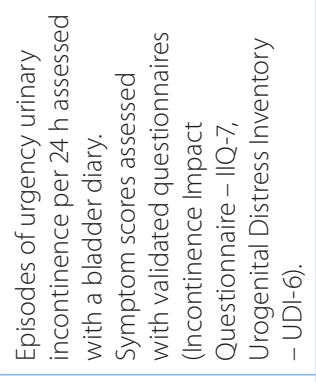 & 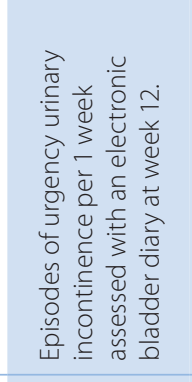 & & & \\
\hline 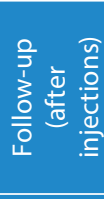 & 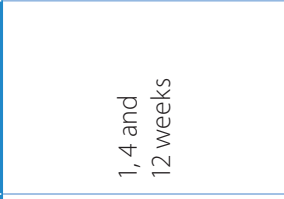 & 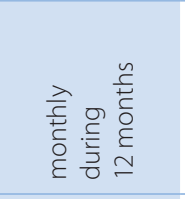 & 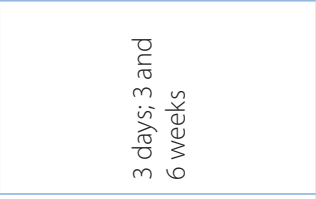 & 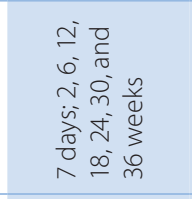 & 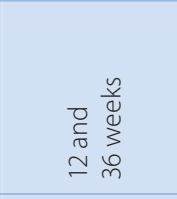 & 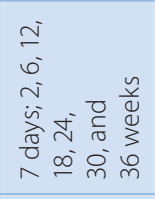 & 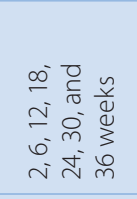 \\
\hline $\begin{array}{l}\stackrel{y}{u} \\
\stackrel{\Delta}{\Delta}\end{array}$ & ? & ? & 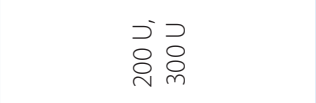 & 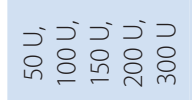 & & & \\
\hline 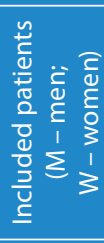 & $\begin{array}{l}\frac{a}{0} \\
\ddot{3} \\
\stackrel{i n}{n} \\
\ddot{\Sigma}\end{array}$ & 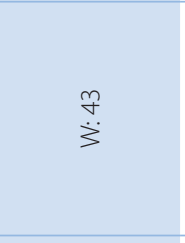 & \begin{tabular}{l}
\multirow{I}{*}{} \\
$\ddot{3}$
\end{tabular} & 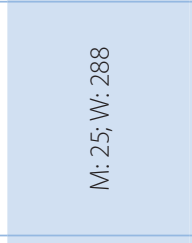 & & & \\
\hline 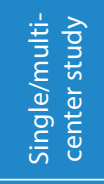 & 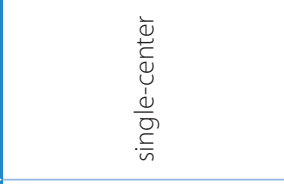 & 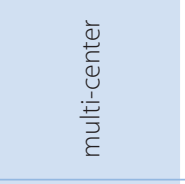 & 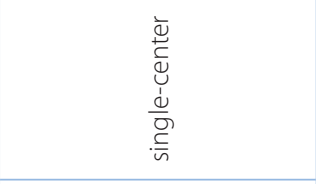 & 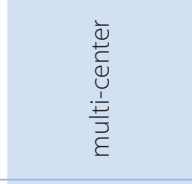 & & & \\
\hline 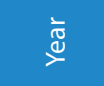 & ڤ্ণे & $\stackrel{\infty}{\stackrel{\infty}{\sim}}$ & ه্ণి & 음 & $\bar{\sigma}$ & $\underset{\sim}{\tilde{N}}$ & $\stackrel{\widetilde{\Sigma}}{\sim}$ \\
\hline 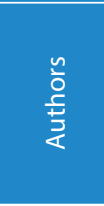 & 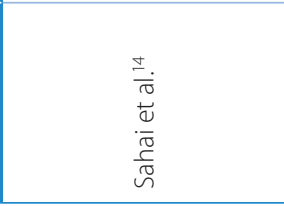 & 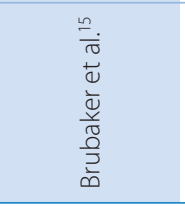 & 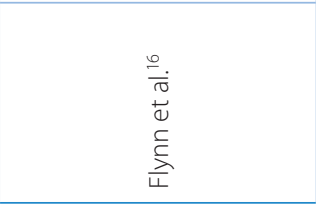 & 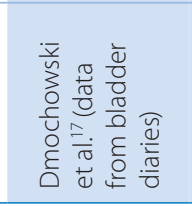 & 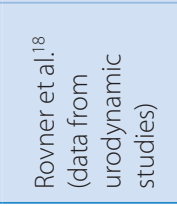 & 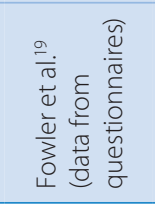 & 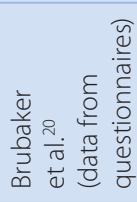 \\
\hline
\end{tabular}




\begin{tabular}{|c|c|c|c|c|c|}
\hline 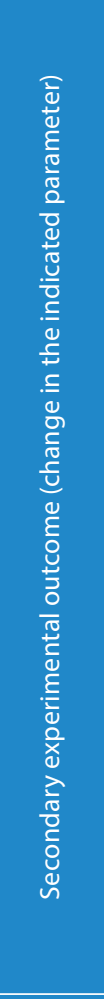 & 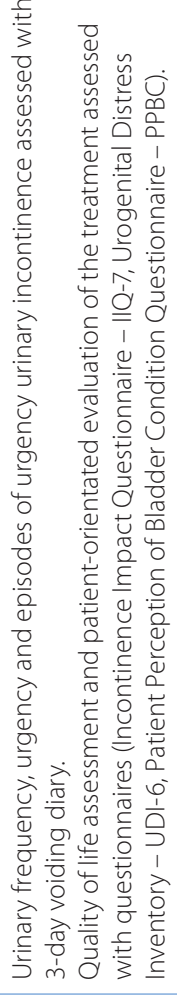 & 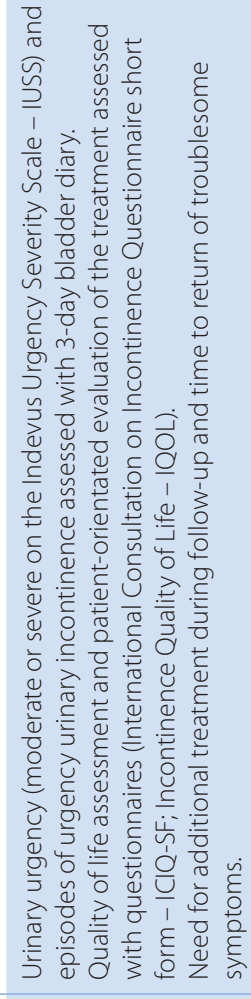 & 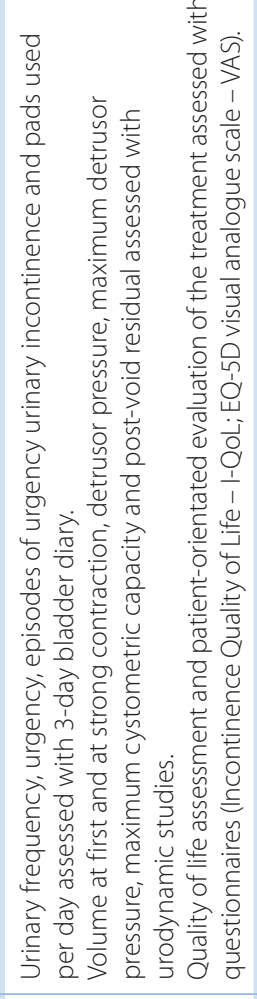 & 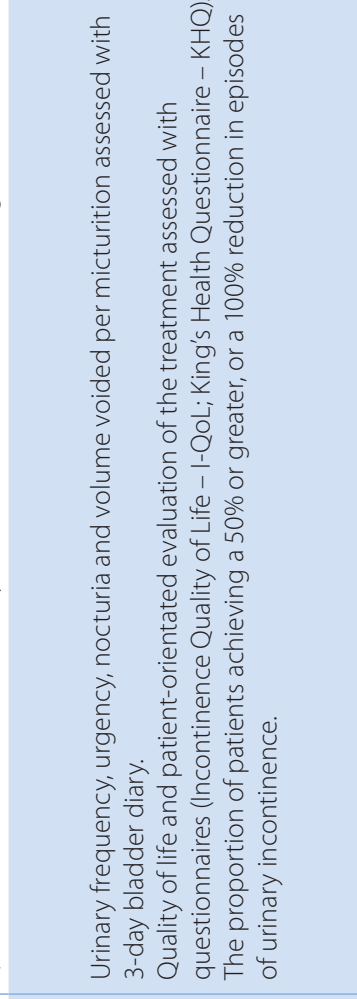 & 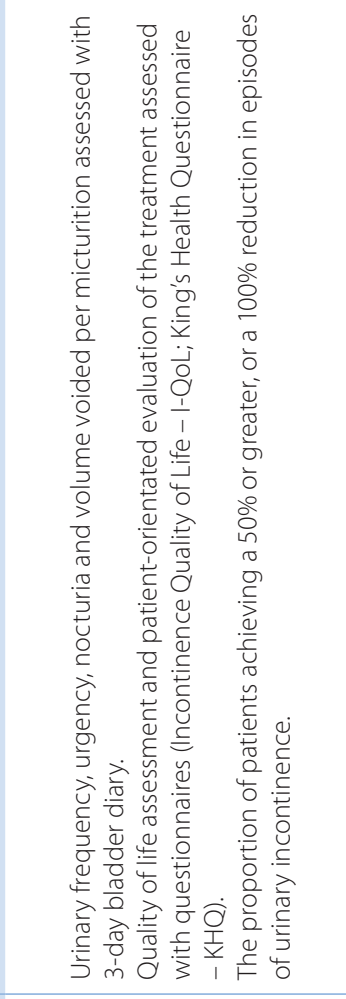 \\
\hline 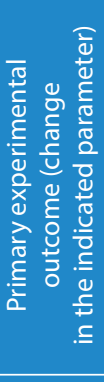 & 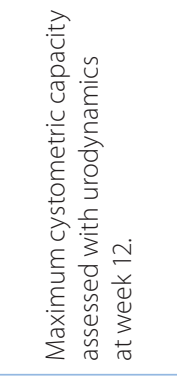 & 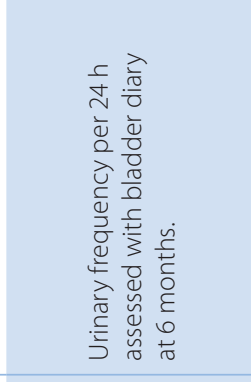 & 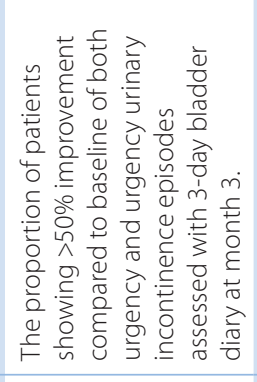 & 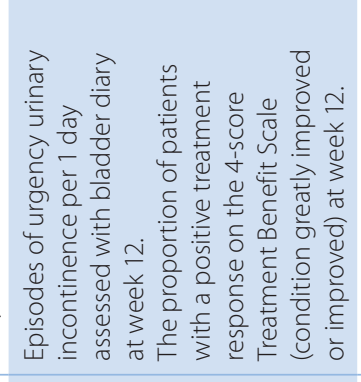 & 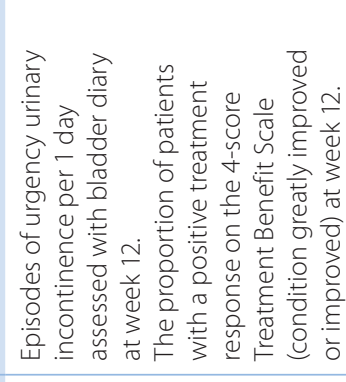 \\
\hline & 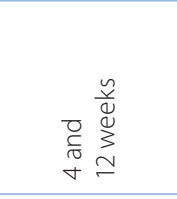 & 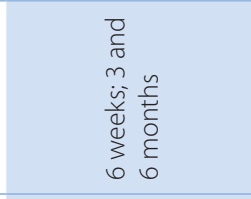 & 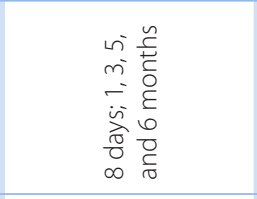 & 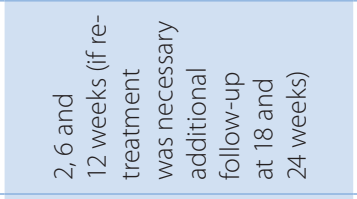 & 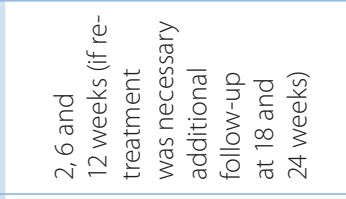 \\
\hline 0 & $\stackrel{?}{\circ}$ & ? & 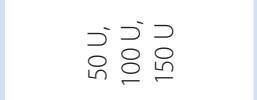 & $\stackrel{?}{\circ}$ & $\stackrel{?}{\circ}$ \\
\hline $\begin{array}{l}\frac{\pi}{0} \\
\frac{0}{0}\end{array}$ & 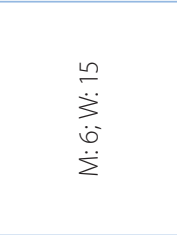 & $\begin{array}{l}\stackrel{9}{+} \\
\stackrel{3}{3}\end{array}$ & 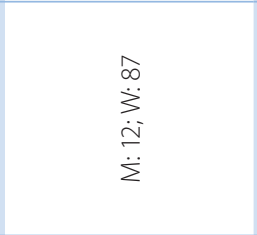 & 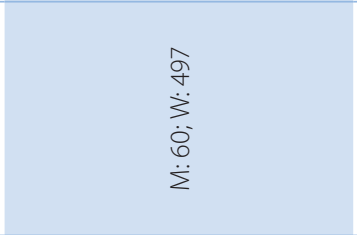 & 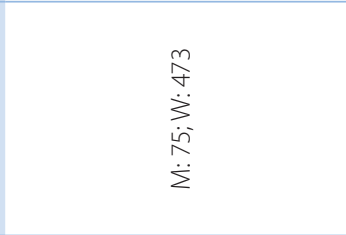 \\
\hline 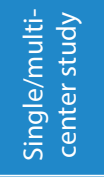 & 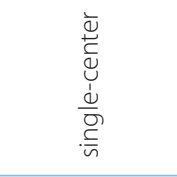 & 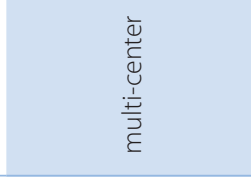 & 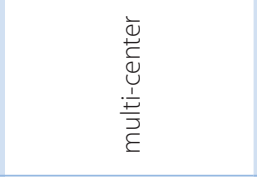 & 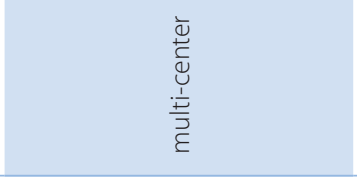 & 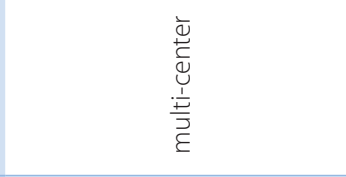 \\
\hline 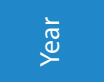 & $\bar{\sigma}$ & $\tilde{\check{~}}$ & స్ & $\bar{\sim}_{\bar{N}}^{m}$ & $\bar{i}$ \\
\hline 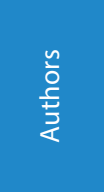 & 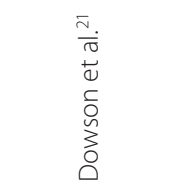 & 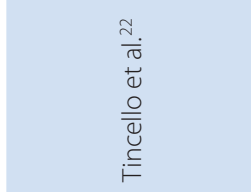 & 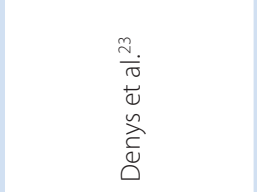 & $\begin{array}{l}\frac{\dot{\sigma}}{\sigma} \\
\frac{U}{U} \\
\dot{E}\end{array}$ & 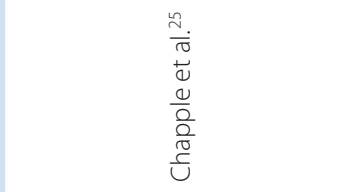 \\
\hline
\end{tabular}




\begin{tabular}{|c|c|c|c|c|c|c|c|c|c|}
\hline 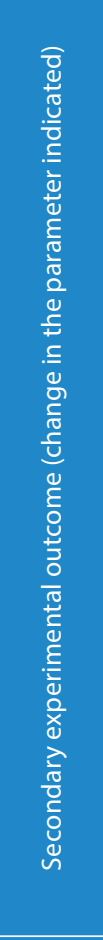 & 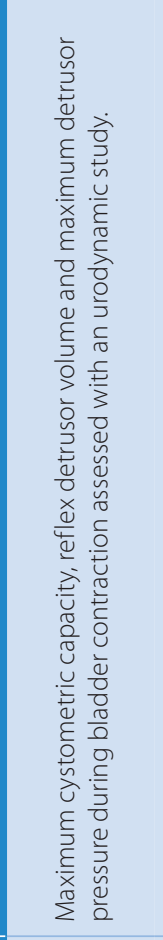 & 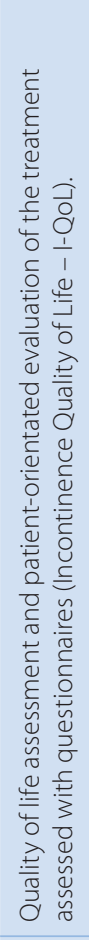 & 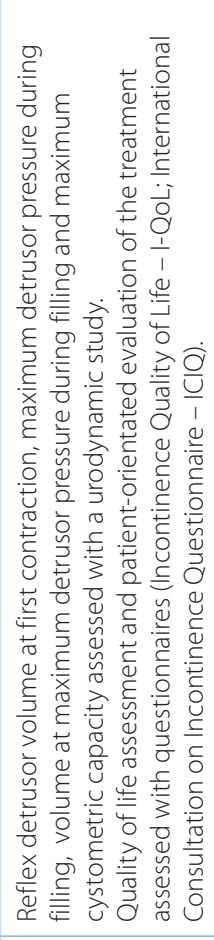 & 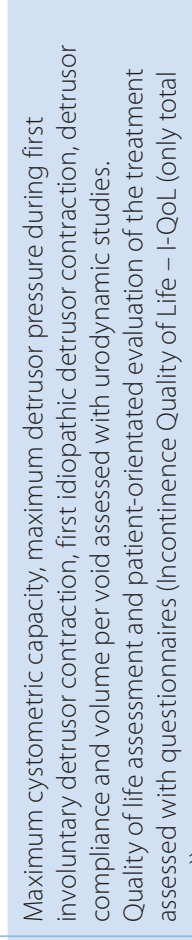 & 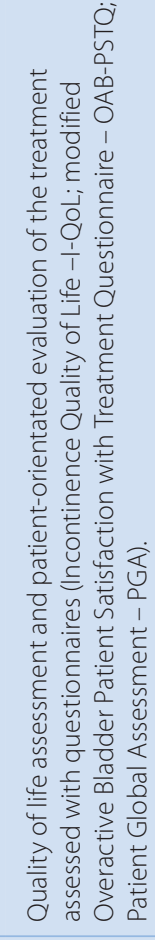 & 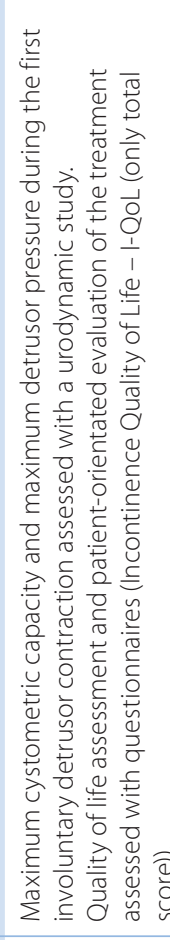 & 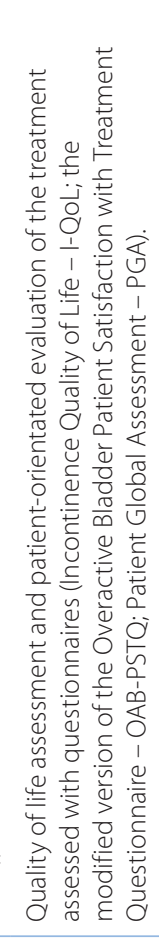 & 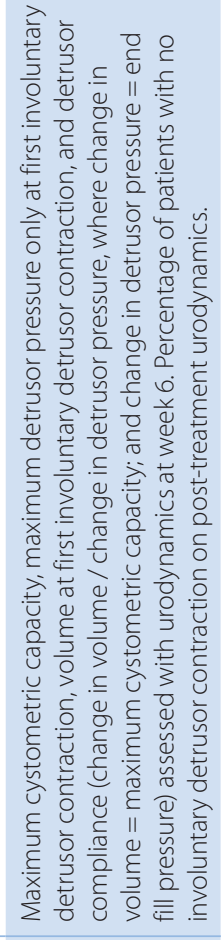 & 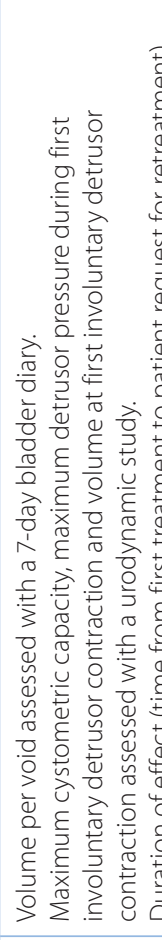 \\
\hline 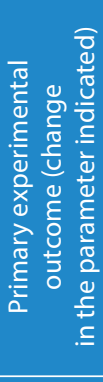 & 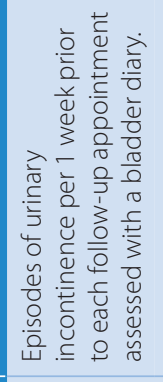 & & 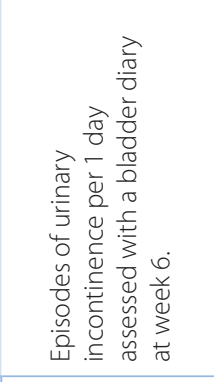 & 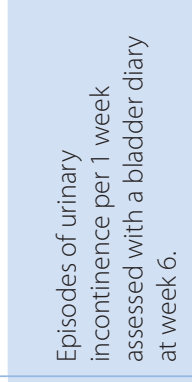 & & 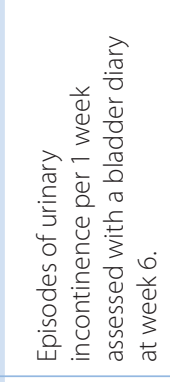 & & & 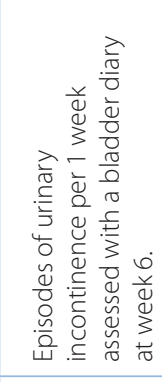 \\
\hline 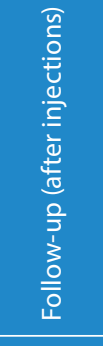 & 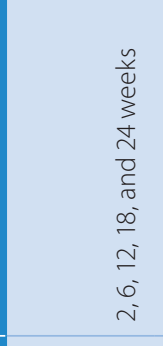 & & 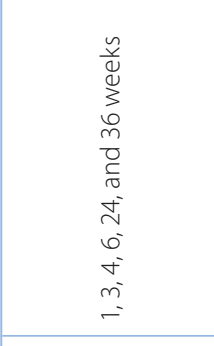 & 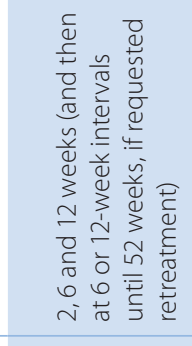 & 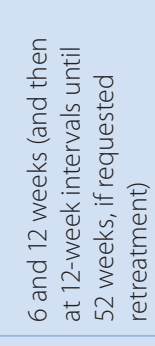 & 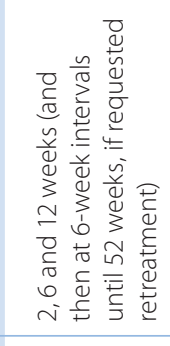 & 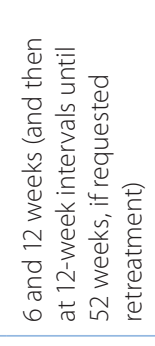 & 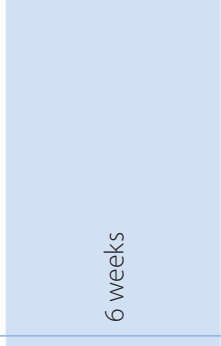 & 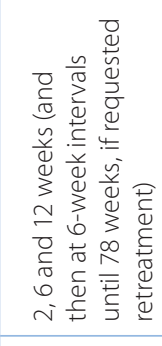 \\
\hline $\begin{array}{l}\text { Uूँ } \\
\stackrel{\Delta}{\Delta}\end{array}$ & $\begin{array}{l}\text { Jे } \\
\text { ठㅇ } \\
\end{array}$ & & 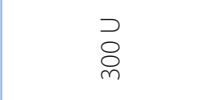 & כొ & & $\begin{array}{l}\text { Jे } \\
\text { o } \\
\text { o }\end{array}$ & & 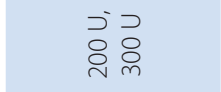 & \\
\hline 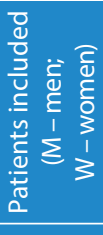 & 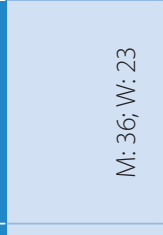 & & 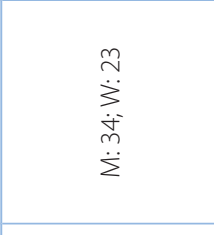 & 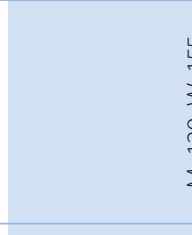 & & & & $\begin{array}{l}8 \\
\dot{+} \\
\ddot{3} \\
\dot{\bar{\alpha}} \\
\\
\ddot{z}\end{array}$ & 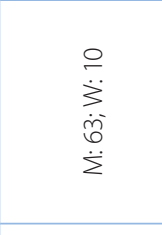 \\
\hline 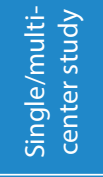 & 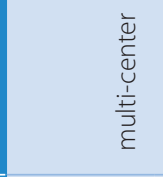 & & 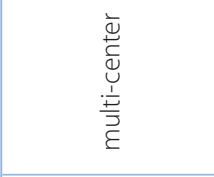 & & & & & 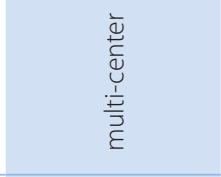 & 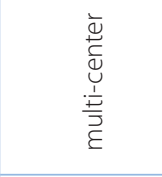 \\
\hline 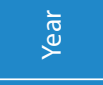 & 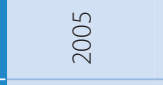 & ڤ్ర & $\overline{\bar{D}}$ & $\overline{\bar{\sigma}}$ & స̃ & $\stackrel{\sim}{\sim}$ & $\stackrel{m}{i}$ & $\stackrel{m}{\bar{i}}$ & $\tilde{\check{\tau}}$ \\
\hline$\frac{5}{\frac{F}{3}}$ & 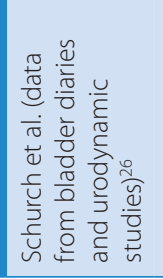 & 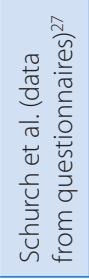 & 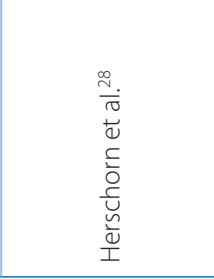 & 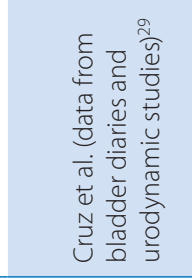 & 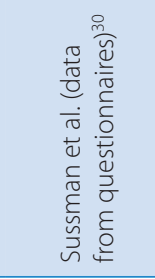 & 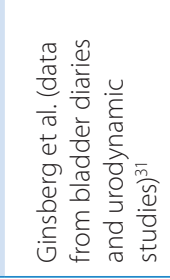 & 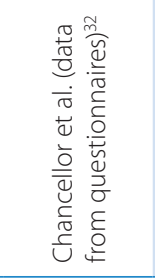 & 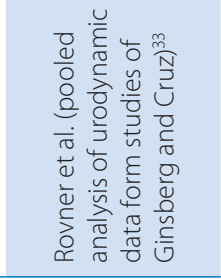 & 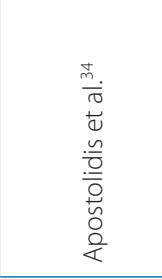 \\
\hline
\end{tabular}


by Dmochowski et al. ( $\mathrm{n}=313$; LE $1, \mathrm{GR}$ B/C), a subgroup analysis for patients with and without detrusor overactivity showed that the patient-reported outcomes were similar in the 2 groups, although statistical significance in comparison with the placebo was not reached in these much smaller subgroups. ${ }^{17}$ Further analysis of the urodynamic data also demonstrated that patients with detrusor overactivity at baseline experienced treatment efficacy compared to those without it. ${ }^{18}$ The authors proposed that urodynamic confirmation of detrusor overactivity before treatment may not be predictive in determining treatment success, as patients with $\mathrm{OAB}$ with and without detrusor overactivity benefited from the treatment. These results are in line with those presented by Schmid et al. from an open-label study ( $\mathrm{n}=100$; LE 2, GR C). ${ }^{35}$ Although the last 2 trials demonstrated some clinical improvement in patients with and without detrusor overactivity, physicians should keep in mind that they were not specifically designed to examine the influence of detrusor overactivity on treatment outcomes and the data presented may be inconsistent.

\section{Maximum detrusor pressure}

There have been $2 \mathrm{OAB}$ studies evaluating the influence of elevated maximum detrusor pressure on final treatment outcomes. Sahai et al., in a subanalysis of urodynamic data from their randomized clinical trial ( $\mathrm{LE} 1, \mathrm{GR} B / \mathrm{C})$, reported that very high maximum detrusor pressure $\left(>110 \mathrm{~cm} \mathrm{H}_{2} \mathrm{O}\right)$ may predict a poor response to treatment with $200 \mathrm{U}$ of onabotulinumtoxinA (sensitivity 0.86 ; specificity 1.0 ), indicating that higher doses may be necessary in these patients. ${ }^{36}$ In turn, Rovner et al. demonstrated that elevated maximum detrusor pressure is a poor indicator of treatment response (LE 1, GR B/C). ${ }^{18}$ Similarly to Sahai et al., their analysis was based on urodynamic data from a previously published randomized clinical trial. It is noteworthy that in the study by Rovner et al., the mean baseline maximum detrusor pressure ranged from $21.7 \mathrm{~cm} \mathrm{H}_{2} \mathrm{O}(\mathrm{SD} \pm 18.26)$ to $24.3 \mathrm{~cm} \mathrm{H}_{2} \mathrm{O}$ ( $\mathrm{SD} \pm 18.40$ ), whereas in the study by Sahai et al. it was much higher, ranging from $74.4 \mathrm{~cm} \mathrm{H}_{2} \mathrm{O}(\mathrm{SD} \pm 32.6)$ to $138.0 \mathrm{~cm} \mathrm{H}_{2} \mathrm{O}(\mathrm{SD} \pm 30.7)$.

In a retrospective analysis of 292 neurogenic patients monitored up to 7 years, high maximum detrusor pressure has been identified as a risk factor of treatment failure. (LE 3, GR C). ${ }^{37}$ Nevertheless, the multivariate analysis failed to confirm it as an independent long-term risk factor.

\section{Maximum cystometric capacity}

A prospective study by Álvares et al. on individuals who had suffered spinal cord injury $(\mathrm{n}=34)$ showed that patients with greater maximum cystometric capacity responded significantly better than others (LE 2, GR B/C). ${ }^{38}$ The authors also demonstrated that cystography parameters, including bladder shape, capacity and the presence of diverticula, were not significantly different between responders and non-responders to the therapy.

\section{Bladder compliance}

It has been suggested that pre-existing bladder wall fibrosis may limit therapeutic efficacy. The impact of bladder compliance on treatment results was assessed by Schmid et al. in their prospective non-randomized study. ${ }^{35}$ A total of 23 men and 77 women with OAB were consecutively treated with injections of $100 \mathrm{U}$ onabotulinumtoxinA and then followed up with urodynamics at 4, 12 and 36 weeks. Treatment failed in 8 patients, all of whom had low bladder compliance (less than $10 \mathrm{~mL} / \mathrm{cm}$ $\mathrm{H}_{2} \mathrm{O}$ ) and maximum cystometric capacity less than $100 \mathrm{~mL}$ due to bladder wall fibrosis (LE 2, GR B). These results are in line with those presented by Kim et al. in neurogenic patients. ${ }^{39}$ Preoperative bladder compliance was significantly lower in non-responders to onabotulinumtoxinA injections $(25.11 \pm 32.59$ vs $8.64 \pm 6.52$; $\mathrm{p}=039)$. Furthermore, a regression analysis revealed that decreased bladder compliance $\left(<10 \mathrm{~mL} / \mathrm{cm} \mathrm{H}_{2} \mathrm{O}\right.$; odds ratio $(\mathrm{OR})=6.041 ; 95 \%$ confidence interval $(\mathrm{CI})=1.189-30.677$; $\mathrm{p}=0.030$ ) was an independent predictor of poor response (LE 3, GR C). A long-term study with a 7-year follow-up of neurogenic patients confirmed in a univariate analysis that decreased bladder compliance is a risk factor for therapy failure (LE 3, GR C). ${ }^{37}$ Moreover, it has been reported that the efficacy of onabotulinumtoxinA injections in patients with low bladder compliance has a shorter duration (12-24 weeks) than in those with normal compliance. ${ }^{40}$

\section{Age}

Komesu et al. compared the efficacy and adverse events of onabotulinumtoxinA injections in women $<65$ and $\geq 65$ years suffering from refractory urgency urinary incontinence (LE 1, GR B). ${ }^{41}$ Even though the authors showed that both older and younger women experienced beneficial reductions in urgency urinary incontinence episodes, similar rates of adverse events and improved quality of life, the study revealed that younger women experienced greater absolute continence, symptom improvement and fewer urinary tract infections. Women $<65$ years had 3.3 -fold greater odds of $\geq 75 \%$ symptom resolution than women $\geq 65$ years $(95 \% \mathrm{CI}=1.56-7.02)$. Compatible results were presented by Cohen et al., but the relationship was not statistically significant in a multivariate analysis (LE 1, GR B). ${ }^{42}$ Lower long-term success rates have also been noted in frail elderly patients (LE 2, GR B). ${ }^{43}$

\section{Sex}

Hsiao et al. showed that male gender is an independent factor associated with OAB treatment failure (LE 2, GR B). ${ }^{44}$ The study included a total of 89 patients ( 46 men 
and 43 women) and found that female gender $(\mathrm{OR}=3.75)$ was an independent factor associated with treatment success.

\section{Symptoms}

It has been shown that low baseline $\mathrm{OAB}$ symptom scores and the presence of $\mathrm{OAB}$ wet are independent factors associated with therapeutic efficacy (LE 2, GR B). ${ }^{44}$ The authors also found that high OAB symptom scores were an independent factor for predicting low response, but the coefficient was low (i.e. -0.12). Thus, symptoms of OAB (other than incontinence) seem to be a poor predictor for assessing efficacy compared to simple evaluation of the presence or absence of incontinence.

\section{Previous anticholinergic treatment}

Makovey et al. reported that patients with poor antimuscarinic efficacy experienced less therapeutic effect from onabotulinumtoxinA injections (LE 3, GR C). ${ }^{45}$ Success rates were lower in patients reporting a lack of efficacy of antimuscarinic drugs ( 34 of $57,68 \%$ ), compared to those who stopped because of side effects ( 24 of $28,86 \%$ ). In contrast, a pooled analysis of 2 trials of $100 \mathrm{U}$ of onabotulinumtoxin A showed no difference in treatment effect irrespective of the number of antimuscarinic preparations tried, or whether oral medication was stopped due to side effects or a lack of efficacy (LE 1, GR B). ${ }^{46}$

\section{Repeated injections}

As the effects of onabotulinumtoxin A therapy are of limited duration, patients usually require further treatments. A recently published study suggested that in $\mathrm{OAB}$ patients who respond to the treatment, the duration of the response declines after the $5^{\text {th }}$ injection, suggesting a possible tolerance effect and a subsequent decline in efficacy (LE 3, GR C) ${ }^{47}$ The mean time between patients receiving intra-detrusor onabotulinumtoxin $A$ and being added to the surgical waiting list for retreatment varied between 8.5 and 10.4 months for the first 5 cycles of treatment, with the longest time period between the $3^{\text {rd }}$ and $4^{\text {th }}$ cycle. It then decreased to 5.5 and 5.25 months between the $5^{\text {th }}$ and $6^{\text {th }}$ cycle and between the $6^{\text {th }}$ and $7^{\text {th }}$ cycle of treatment, respectively. Further data extrapolation has shown that at the $9^{\text {th }}$ or $10^{\text {th }}$ treatment, the mean duration of response to the treatment would be less than the recommended 4 months. The authors also proposed that treatments with symptomatic benefit of less than 3 months should be regarded as "treatment failure", i.e., patients who undergo further treatments with onabotulinumtoxin A may not get a sufficient therapeutic benefit to justify repeating the procedure. These results are similar to those presented by Tincello et al., who found an increased rate of symptom recurrence following $2^{\text {nd }}$ and $3^{\text {rd }}$ treatments when compared to symptom recurrence after the $1^{\text {st }}$ treatment (LE 3, GR C). ${ }^{48}$ Marcelissen et al. showed that almost $70 \%$ of all patients abandoned repeated treatments after a mean follow-up of 97 months (LE 3, GR C). ${ }^{49}$ Of the patients included ( $\left.\mathrm{n}=128\right), 27 \%$ experienced insufficient effect and $43 \%$ had tolerance issues.

There have been 2 studies investigating the efficacy of repeated injections in neurogenic individuals in terms of risk factors for treatment failure. A recent study by Denys et al. have showed that patients with NDO with $\mathrm{a} \geq 50 \%$ reduction of urinary incontinence after their $1^{\text {st }}$ onabotulinumtoxin A treatment continued to experience consistent improvements in urinary incontinence and quality of life with subsequent treatments over a period of 4 years (LE 2, GR B). ${ }^{50}$ Initial positive responses were generally maintained to the same extent after repeat treatments. A $<50 \%$ reduction of urinary incontinence after the $1^{\text {st }}$ treatment did not necessarily predict low response with subsequent treatments, as more than $1 / 3$ of the patients with poor initial response showed better results and increasing response with subsequent injections. The authors concluded that these results underscore the importance of attempting at least a $2^{\text {nd }}$ treatment with onabotulinumtoxinA before deeming neurogenic patients unsuitable for this therapy. Joussain et al. in their retrospective analysis of 292 patients, reported a failure rate of $12.6 \%(8.6-16.5 \%)$ after 3 years, $22.2 \%(16.6-27.3 \%)$ after 5 years and $28.9 \%$ (21.9-35.3\%) after 7 years of follow-up, whereas the primary failure ratio was $5.1 \%(\mathrm{n}=15 ; \mathrm{LE} 3, \mathrm{GR} C) .{ }^{37}$

\section{Other factors}

In neurogenic patients, other possible risk factors that may predict treatment failure include a high number of febrile urinary tract infections (LE 3, GR C) and kidney ultrasound abnormalities (LE 3, GR C). ${ }^{37}$

In $\mathrm{OAB}$ patients, smoking status has been found to be a predictive factor for non-response in urgency episodes (with smokers having nearly 3 times greater odds of nonresponse compared to non-smokers), and higher numbers of baseline leakage episodes are correlated with failure to achieve continence (for every additional increase in the number of baseline leakage episodes, patients had a $17 \%$ increase in the odds of failing to achieve continence; LE 1, GR B). ${ }^{51}$ Body mass index (BMI) has been shown to have marginal associations with non-response to the treatment. It is worth noting that the risk factors identified in the study were derived from OAB women treated with $200 \mathrm{U}$ of onabotulinumtoxinA and urodynamically proven detrusor overactivity. Furthermore, the authors assessed potential patient factors correlated with non-response only at 6 weeks after treatment. Urinary tract infections in $\mathrm{OAB}$ patients were not found to affect the outcomes of the treatment (LE 1, GR B). ${ }^{51}$ 


\section{Predictive factors for $\mathrm{CIC}$}

There are no universally accepted predictive factors for the need for CIC following injections of onabotulinumtoxin A. However, the reported rates of CIC use seem to depend on the etiology of detrusor overactivity, the injected dose along with the injection technique, the definition of significant post-void residual urine, and other factors. It has been shown that the risk of post-treatment retention requiring $\mathrm{CIC}$ can be 2 -fold higher in neurogenic patients than in those with OAB (LE 2, GR B). ${ }^{52}$ The affected neural mechanism for voiding in neurogenic individuals has been proposed as a reason for this phenomenon. The majority of available studies observed a dose-response relationship in terms of initiating $\mathrm{CIC}$ following onabotulinumtoxin $\mathrm{A}$ injections (LE 1, GR A). ${ }^{23,26,35}$ Currently recommended doses (200 U in NDO, $100 \mathrm{U}$ in OAB) represent an appropriate balance between the benefits and safety profile of the drug. A meta-analysis of efficacy and adverse events after trigonal vs extratrigonal injections revealed that trigonal injections were more often associated with acute urinary retention, but this correlation was not statistically significant. ${ }^{53}$ Trigonal injections also lead to non-significantly higher values of post-void residual urine. The post-void residual (PVR) volume at which patient should start $\mathrm{CIC}$ varies among the available studies. Proposed cut-offs include $100 \mathrm{~mL}$, $150 \mathrm{~mL}, 200 \mathrm{~mL}$, or even $300 \mathrm{~mL} .{ }^{14,15,35,52,54}$ To make matters even more complex, the symptoms associated with retention have not always been included in the study definitions of significant retention. There are no strict criteria for CIC cessation, thus the duration of CIC may have been overestimated in some studies. Finally, a recent study has shown that CIC initiated on the basis of an arbitrary PVR volume does not benefit the patient, and CIC use should be based on the individual patient's symptoms (LE 3, GR B/C). ${ }^{55}$ Nevertheless, a peak effect and dose-dependent mean increase in PVR volume following onabotulinumtoxinA treatment is usually observed at week 2 , with a gradual decrease between weeks 4 and 12 (LE 1, GR A). ${ }^{17,24}$ Furthermore, the duration of catheterization (by CIC or indwelling catheter) is typically longer with higher injected doses. ${ }^{17}$ The need for CIC also depends on the patient's medical history of previous injections. On the one hand, patients who do not need CIC after their first injection are at a lower risk of needing CIC in later treatment cycles (LE 1, GR A). ${ }^{56}$ On the other hand, clinicians should remember that if $\mathrm{CIC}$ is necessary after the first injection, it seems to be needed after all subsequent treatments (LE 2, GR B). ${ }^{54}$ In patients with a preoperative PVR volume $>100 \mathrm{~mL}$, retention may appear in up to $95 \%$ of patients (LE 3, GR C). ${ }^{57}$ Similar findings have been demonstrated in terms of low voiding efficiency ( $<90 \%$ of the voided volume compared to the pre-void bladder volume) (LE 3, GR C). ${ }^{58}$ Furthermore, it has been shown that patients with low maximum flow rates $(<15 \mathrm{~mL} / \mathrm{s})$, low projected isovolumetric pressure $(<50$, calculated as the maximum urinary flow rate
+ detrusor pressure at the maximum urinary flow rate in women) and low bladder contractility index $(<120$, calculated as 5 times the maximum urinary flow rate + detrusor pressure at the maximum urinary flow rate in men) are at increased risk of needing CIC after onabotulinumtoxinA injections (LE 3, GR C). ${ }^{59}$ Clean intermittent catheterization may also become necessary after later injections even if it was not initially required. Other factors that may increase the risk of CIC include older age ( $>61-76$ years) (LE 2, GR B), ${ }^{43,60}$ frailty (LE 2, GR B) ${ }^{43}$ and higher parity (particularly a higher number of vaginal deliveries; LE 2, GR B). ${ }^{60}$ A recent study has shown that BMI and concomitant comorbidities do not significantly influence PVR volumes and the risk of CIC, even though one would expect that these factors could provoke safety issues. ${ }^{60}$ Nevertheless, Wang et al. showed that patients with diabetes have a significantly increased incidence of PVR volumes greater than $150 \mathrm{~mL}$ (60.4\%) compared with nondiabetic patients (33.3\%; LE 3, GR C). ${ }^{61}$

Although it may sometimes be necessary to introduce CIC, studies have shown that the need to perform CIC do not negatively impact the outcome of onabotulinumtoxin $\mathrm{A}$ therapy, and that improvements in patients' symptoms are similar with and without CIC. ${ }^{31,57}$ It has been shown that the risk of new-onset urine retention with a need for CIC usually disappears 2 weeks after the injections. ${ }^{60}$

\section{Discussion}

To the best of our knowledge, this is the first study summarizing reported methods of efficacy assessment and factors predicting failure in onabotulinumtoxin A therapy. Currently, onabotulinumtoxinA is the only formulation of botulinum toxin that has been investigated in properly powered, multicenter, multinational randomized controlled trials, and only onabotulinumtoxin A is licensed in the USA and Europe for the management of NDO and OAB.

Our study revealed that most of the randomized controlled trials investigating the efficacy of intra-detrusor injections with onabotulinumtoxin $\mathrm{A}$, both in $\mathrm{OAB}$ and NDO patients, commonly used the number of incontinence episodes as the primary experimental outcome. However, this parameter cannot be directly applied in daily clinical practice, as it is not always well correlated with subjective outcomes, including health-related quality of life, treatment satisfaction, subjective assessments of global "improvement", or the safety of the upper urinary tract. ${ }^{20}$

As $\mathrm{OAB}$ is a syndrome in the absence of urinary tract infection or other obvious pathology, outcomes from treatment are subjective, highly individual and influenced by patients' lifestyle as well as their expectations from treatment. As the patient's perception of treatment success in OAB is an important component of overall success, clinicians should keep in mind that they underestimate the extent to which patients are affected by their symptoms 
in $25-37 \%$ of cases. ${ }^{62}$ Therefore, reports of medical outcomes after treatment should always include independent, validated questionnaires self-administered by patients to avoid interviewer bias. ${ }^{63}$ Furthermore, it has been demonstrated that objective measures from bladder diaries have correlated poorly with patient-related outcome measures, suggesting that objective and subjective assessments measure different aspects of treatment efficacy. ${ }^{64}$ Therefore, any evaluation of the efficacy of intra-detrusor injections with onabotulinumtoxinA in day-to-day clinical treatment of OAB patients should be based on objective measures (from bladder diaries) as well as patient-reported outcomes (from questionnaires). The International Consultation on Incontinence has evaluated specific criteria for currently used questionnaires and developed a recommendation grading system. Questionnaires with a Grade A recommendation (highly recommended) should be used in clinical practice. Among them are the Overactive Bladder Questionnaire $(\mathrm{OAB}-\mathrm{q})$, the Overactive Bladder Satisfaction Questionnaire (OAB-S), the Overactive Bladder Symptom Scores Questionnaire (OABSS), the Incontinence Impact Questionnaire (II-Q), and the Urogenital Distress Inventory (UDI). Nowadays, single-question questionnaires (e.g., the Patient Global Impression Scale, the Treatment Benefit Scale, the Likert scale, and VAS score ${ }^{65}$ ) are becoming more popular and are widely used in daily practice. The lack of a positive change in these scales following treatment could be used to define treatment failure. Each questionnaire can be used alone or in combination with other questionnaires to improve assessment or monitoring of treatment outcomes. ${ }^{4}$ Some experts suggest that in daily clinical practice, treatment outcomes in OAB patients can be judged by patient communication regarding changes in their symptoms or improvements in their quality of life, without objective documentation. In our opinion, this practice should be avoided. Assessment of treatment efficacy with urodynamics in $\mathrm{OAB}$ patients is not currently recommended, as a recently published systematic review with meta-analysis has shown that urodynamic parameters may not differ despite significant improvement in patientoriented outcomes. ${ }^{2}$ Current guidelines advise that followup should be patient/symptom directed and a new injection may be considered when the clinical benefit of the previous injection diminishes, but a period of 3 months must elapse between each injection. ${ }^{66}$ Also, the manufacturer of onabotulinumtoxin A recommends that retreatment should not be considered within 12 weeks from the previous bladder injection, and the total dose should not exceed $360 \mathrm{U}$ in a 3 -month period for all indications. ${ }^{67}$

There are no guidelines or recommendations available for following up neurogenic patients treated with intradetrusor onabotulinumtoxinA injections. As neurological diseases are usually progressive, videourodynamic study and the use of validated questionnaires with a bladder diary at baseline are recommended. The primary goal of bladder management in neurogenic patients is to achieve low-pressure urine storage and low-pressure emptying of the bladder. Thus, the therapy should be assessed with a urodynamic study, even though available randomized controlled trails used changes in the frequency of urinary incontinence episodes as a primary experimental outcome. In high-risk patients (i.e., those with vesicoureteral reflux, elevated detrusor pressures, decreased bladder compliance, and worsening hydronephrosis or renal function), experts recommend repeating urodynamic studies after injections regardless of the clinical outcome. ${ }^{68}$ In the current literature, there is a tendency to define unsuccessful treatment with maximum storage pressure $>40 \mathrm{~cm} \mathrm{H}_{2} \mathrm{O}$ and/or detrusor compliance $<20 \mathrm{~mL} / \mathrm{cm} \mathrm{H}_{2} \mathrm{O}$ after injections. Moreover, it has been proposed that the following parameters should be recorded: the presence of involuntary detrusor contractions, the volume of bladder filling at the first involuntary contraction, the maximum detrusor pressure of involuntary contractions of greater intensity, the maximum cystometric capacity, the first voiding desire, bladder compliance, maximum detrusor pressure, maximum flow, detrusor pressure at maximum flow, and post-void residual volume. ${ }^{69}$ In low-risk patients (i.e., those with low detrusor storage pressures and appropriate compliance, and when other clinical evaluations also suggest stable lower and upper urinary tracts in a non-progressive neurologic disease), urodynamic studies after injections can be delayed if the patient responds clinically to the treatment. ${ }^{68}$

Our study revealed that possible risk factors that may influence treatment outcomes include urodynamically proven detrusor overactivity (LE 1, GR D), elevated maximum detrusor pressure (LE $1, G R B / C)$, greater maximum cystometric capacity (LE 2, GR B/C), impaired bladder compliance (LE 2, GR B), older age (LE 1, GR B), male gender (LE 2, GR B), higher bother score from symptoms at baseline (LE 2, GR B), previous anticholinergic treatment (LE 3, GR D), repeated injections with a subsequent decline in efficacy (LE 3, GR C), and other factors (LE 2/3, GR B/C). Patients with these risk factors should be informed that they may experience less efficacy. It might be suggested that the predictors of success identified by our study may vary depending on treatment dose, but it has recently been emphasized that there is no physiological or pharmacological reason why such a difference would exist. ${ }^{51}$

In patients who did not respond to the treatment, other potential underlying disorders for the symptoms reported should be taken into account. It is noteworthy that patients with $O A B$ symptoms may in fact suffer from painful bladder or interstitial cystitis; these individuals appear to respond less favorably to with onabotulinumtoxin $\mathrm{A},{ }^{70}$ and if the treatment is effective, it has a shorter duration of action. ${ }^{71}$ In patients with decreased bladder compliance, special attention should be given to any possible underlying neurological disorders, as impaired compliance is usually a sequela of neurologic disease, but it may also result from any process that destroys the elastic or viscoelastic properties of the bladder wall. In neurogenic patients, failed 
treatment may also result from progression of their underlying neurological disorder. Physicians should remember that short duration of efficacy or treatment failure can be dependent upon procedure-related factors, such as problems with the storage of onabotulinumtoxinA vials, the reconstitution process or the injection technique. Optimization of the injection procedure (the first indication; dose personalization depending on clinical and urodynamic parameters, but also patient expectations; injection localization) could help improve the global efficacy of onabotulinumtoxinA treatment. Moreover, associated urinary tract infections can mask effective treatment. Development of neutralizing antibodies against the neurotoxin is possible, but very unlikely. It has been demonstrated that $<1 \%$ of patients develop neutralizing antibodies to onabotulinumtoxinA after treatment cycle 6 and their presence has no significant impact on treatment efficacy. ${ }^{23,72}$ Another hypothesis to explain treatment failure could be histological modifications induced by injections. However, studies have demonstrated no difference in terms of inflammation, fibrosis or edema before and after 1 or multiple injections. ${ }^{73}$ Moreover, no histological differences were found between responders and non-responders. Patients for whom the treatment failed should be questioned with regard to changes in lifestyle or the use of new medications that may have contributed to the worsening of lower urinary tract symptoms.

If the treatment fails and/or a physician predicts that intra-detrusor injections of onabotulinumtoxin A might be ineffective, a switch to a different toxin seems to be more effective than administering another injection of the same toxin. Replacing onabotulinumtoxinA with abobotulinumtoxin A may provide satisfying results. ${ }^{74}$ It has been also proposed that in non-responding patients planned for repeated injections, the dose may be increased by an extra $50 \mathrm{U}$ in OAB and an extra $100 \mathrm{U}$ in NDO individuals. ${ }^{69}$

Some studies describe a phenomenon called "secondary failure". This term is used for patients who responded well to the first injection(s), but eventually had very limited benefit after subsequent treatments. Possible causes include an underlying immunological mechanism (antibody formation) or a technical issue with subsequent injections (less adequate delivery). Therefore, some experts have proposed that a repeat injection at least 3 months after the failed injection should be performed in patients with secondary failure. ${ }^{75}$

\section{Conclusions}

In conclusion, onabotulinumtoxin $\mathrm{A}$ is well established as a second-line treatment for patients with $\mathrm{OAB}$ and NDO. When following up OAB patients treated with onabotulinumtoxin A, a validated questionnaire should be used to measure the degree of symptom bother, while a voiding diary should be utilized to assess the response to the treatment. In neurogenic individuals, the therapy should be also evaluated with a urodynamic study. Patients with risk factors for treatment failure should be appropriately informed of the possible lack of efficacy. This article can serve as a reference document for future research assessing the efficacy of onabotulinumtoxinA in specific clinical situations and help urologists select appropriate patients and evaluate their response to the treatment.

\section{References}

1. Olivera CK, Meriwether K, El-Nashar S, et al; Systematic Review Group for the Society of Gynecological Surgeons. Nonantimuscarinic treatment for overactive bladder: A systematic review. Am J Obstet Gynecol. 2016;215(1):34-57.

2. Sun Y, Luo D, Tang C, Yang L, Shen H. The safety and efficiency of onabotulinumtoxinA for the treatment of overactive bladder: A systematic review and meta-analysis. Int Urol Nephrol. 2015;47(11):1779-1788.

3. Cui Y, Zhou X, Zong H, Yan H, Zhang Y. The efficacy and safety of onabotulinumtoxinA in treating idiopathic OAB: A systematic review and meta-analysis. Neurourol Urodyn. 2015;34(5):413-419.

4. Corcos J, Przydacz M, Campeau L, et al. CUA guideline on adult overactive bladder. Can Urol Assoc J. 2017;11(5):E142-E173.

5. Zhang R, Xu Y, Yang S, Liang H, Zhang Y, Liu Y. OnabotulinumtoxinA for neurogenic detrusor overactivity and dose differences: A systematic review. Int Braz J Urol. 2015;41(2):207-219.

6. Zhou X, Yan HL, Cui YS, Zong HT, Zhang Y. Efficacy and safety of onabotulinumtoxinA in treating neurogenic detrusor overactivity: A systematic review and meta-analysis. Chin Med J (Engl). 2015;128(7):963-968.

7. Yuan H, Cui Y, Wu J, Peng P, Sun X, Gao Z. Efficacy and adverse events associated with use of onabotulinumtoxinA for treatment of neurogenic detrusor overactivity: A meta-analysis. Int Neurourol J. 2017;21(1):53-61.

8. Cheng T, Shuang WB, Jia DD, et al. Efficacy and safety of onabotulinumtoxinA in patients with neurogenic detrusor overactivity: A systematic review and meta-analysis of randomized controlled trials. PLoS One. 2016;11(7):e0159307.

9. Anger JT, Weinberg A, Suttorp MJ, Litwin MS, Shekelle PG. Outcomes of intravesical botulinum toxin for idiopathic overactive bladder symptoms: A systematic review of the literature. J Urol. 2010;183(6):2258-2264.

10. Karsenty G, Denys P, Amarenco G, et al. Botulinum toxin A (Botox) intradetrusor injections in adults with neurogenic detrusor overactivity/neurogenic overactive bladder: A systematic literature review. Eur Urol. 2008;53(2):275-287.

11. Soljanik I. Efficacy and safety of botulinum toxin A intradetrusor injections in adults with neurogenic detrusor overactivity/neurogenic overactive bladder: A systematic review. Drugs. 2013;73(10): 1055-1066.

12. University of Oxford, Graduate School in EBM and Research Methods, Centre for Evidence-Based Medicine, Oxford Centre for EvidenceBased Medicine. Levels of Evidence and Grades of Recommendation. 2009. http://www.cebm.net/oxford-centre-evidence-basedmedicine-levels-evidence-march-2009/. Accessed January 11, 2017.

13. Milsom I, Abrams P, Cardozo L, Roberts RG, Thüroff J, Wein AJ. How widespread are the symptoms of an overactive bladder and how are they managed? A population-based prevalence study. BJU Int. 2001;87(9):760-766.

14. Sahai A, Khan MS, Dasgupta P. Efficacy of botulinum toxin-A for treating idiopathic detrusor overactivity: Results from a single center, randomized, double-blind, placebo controlled trial. J Urol. 2007;177(6):2231-2236.

15. Brubaker L, Richter HE, Visco A, et al; Pelvic Floor Disorders Network. Refractory idiopathic urge urinary incontinence and botulinum A injection. J Urol. 2008;180(1):217-222.

16. Flynn MK, Amundsen CL, Perevich M, Liu F, Webster GD. Outcome of a randomized, double-blind, placebo controlled trial of botulinum A toxin for refractory overactive bladder. J Urol. 2009;181(6):2608-2615.

17. Dmochowski R, Chapple C, Nitti VW, et al. Efficacy and safety of onabotulinumtoxinA for idiopathic overactive bladder: A doubleblind, placebo controlled, randomized, dose ranging trial. $J$ Urol. 2010;184(6):2416-2422. 
18. Rovner E, Kennelly M, Schulte-Baukloh H, Zhou J, Haag-Molkenteller C, Dasgupta P. Urodynamic results and clinical outcomes with intradetrusor injections of onabotulinumtoxinA in a randomized, placebo-controlled dose-finding study in idiopathic overactive bladder. Neurourol Urodyn. 2011;30(4):556-562.

19. Fowler CJ, Auerbach S, Ginsberg D, et al. OnabotulinumtoxinA improves health-related quality of life in patients with urinary incontinence due to idiopathic overactive bladder: A 36-week, doubleblind, placebo-controlled, randomized, dose-ranging trial. Eur Urol. 2012;62(1):148-157.

20. Brubaker L, Gousse A, Sand P, et al. Treatment satisfaction and goal attainment with onabotulinumtoxinA in patients with incontinence due to idiopathic OAB. Int Urogynecol J. 2012;23(8):1017-1025.

21. Dowson C, Sahai A, Watkins J, Dasgupta P, Khan MS. The safety and efficacy of botulinum toxin-A in the management of bladder oversensitivity: A randomized double-blind placebo-controlled trial. Int J Clin Pract. 2011;65(6):698-704.

22. Tincello DG, Kenyon S, Abrams KR, et al. Botulinum toxin a versus placebo for refractory detrusor overactivity in women: A randomized blinded placebo-controlled trial of 240 women (the RELAX study). Eur Urol. 2012;62(3):507-514.

23. Denys P, Le Normand L, Ghout I, et al; VESITOX study group in France. Efficacy and safety of low doses of onabotulinumtoxinA for the treatment of refractory idiopathic overactive bladder: A multicenter, double-blind, randomized, placebo-controlled dose-ranging study. Eur Urol. 2012;61(3):520-529.

24. Nitti VW, Dmochowski R, Herschorn S, et al; EMBARK Study Group. OnabotulinumtoxinA for the treatment of patients with overactive bladder and urinary incontinence: Results of a phase 3, randomized, placebo controlled trial. J Urol. 2013;189(6):2186-2193.

25. Chapple C, Sievert KD, MacDiarmid S, et al. OnabotulinumtoxinA $100 \mathrm{U}$ significantly improves all idiopathic overactive bladder symptoms and quality of life in patients with overactive bladder and urinary incontinence: A randomized, double-blind, placebo-controlled trial. Eur Urol. 2013;64(2):249-256.

26. Schurch B, de Seze M, Denys $P$, et al; Botox Detrusor Hyperreflexia Study Team. Botulinum toxin type a is a safe and effective treatment for neurogenic urinary incontinence: Results of a single treatment, randomized, placebo controlled 6-month study. J Urol. 2005;174(1):196-200.

27. Schurch B, Denys P, Kozma CM, Reese PR, Slaton T, Barron RL. Botulinum toxin $A$ improves the quality of life of patients with neurogenic urinary incontinence. Eur Urol. 2007;52(3):850-858.

28. Herschorn S, Gajewski J, Ethans K, et al. Efficacy of botulinum toxin A injection for neurogenic detrusor overactivity and urinary incontinence: A randomized, double-blind trial. J Urol. 2011;185(6):2229-2235.

29. Cruz F, Herschorn S, Aliotta P, et al. Efficacy and safety of onabotulinumtoxinA in patients with urinary incontinence due to neurogenic detrusor overactivity: A randomized, double-blind, placebo-controlled trial. Eur Urol. 2011;60(4):742-750.

30. Sussman D, Patel V, Del Popolo G, Lam W, Globe D, Pommerville P. Treatment satisfaction and improvement in health-related quality of life with onabotulinumtoxinA in patients with urinary incontinence due to neurogenic detrusor overactivity. Neurourol Urodyn. 2013;32(3):242-249.

31. Ginsberg D, Gousse A, Keppenne V, et al. Phase 3 efficacy and tolerability study of onabotulinumtoxinA for urinary incontinence from neurogenic detrusor overactivity. J Urol. 2012;187(6):2131-2139.

32. Chancellor MB, Patel V, Leng WW, et al. OnabotulinumtoxinA improves quality of life in patients with neurogenic detrusor overactivity. Neurology. 2013;81(9):841-848.

33. Rovner E, Dmochowski R, Chapple C, Thompson C, Lam W, HaagMolkenteller C. OnabotulinumtoxinA improves urodynamic outcomes in patients with neurogenic detrusor overactivity. Neurourol Urodyn. 2013;32(8):1109-1115.

34. Apostolidis A, Thompson C, Yan X, Mourad S. An exploratory, placebo-controlled, dose-response study of the efficacy and safety of onabotulinumtoxinA in spinal cord injury patients with urinary incontinence due to neurogenic detrusor overactivity. World J Urol. 2013;31(6):1469-1474.

35. Schmid DM, Sauermann P, Werner M, et al. Experience with 100 cases treated with botulinum-A toxin injections in the detrusor muscle for idiopathic overactive bladder syndrome refractory to anticholinergics. J Urol. 2006;176(1):177-185.
36. Sahai A, Khan MS, Le Gall N, Dasgupta P; GKT Botulinum Study Group. Urodynamic assessment of poor responders after botulinum toxin-A treatment for overactive bladder. Urology. 2008;71(3):455-459.

37. Joussain $C$, Popoff $M$, Phe $V$, et al. Long-term outcomes and risks factors for failure of intradetrusor onabotulinumtoxin $A$ injections for the treatment of refractory neurogenic detrusor overactivity. Neurourol Urodyn. 2017;37(2):799-806.

38. Alvares RA, Araujo ID, Sanches MD. A pilot prospective study to evaluate whether the bladder morphology in cystography and/or urodynamic may help predict the response to botulinum toxin-A injection in neurogenic bladder refractory to anticholinergics. BMC Urol. 2014;14:66.

39. Kim SW, Choi JH, Lee YS, Han SW, Im YJ. Preoperative urodynamic factors predicting outcome of botulinum toxin-A intradetrusor injection in children with neurogenic detrusor overactivity. Urology. 2014;84(6):1480-1484.

40. Klaphajone J, Kitisomprayoonkul W, Sriplakit S. Botulinum toxin type A injections for treating neurogenic detrusor overactivity combined with low-compliance bladder in patients with spinal cord lesions. Arch Phys Med Rehabil. 2005;86(11):2114-2118.

41. Komesu YM, Amundsen CL, Richter HE, et al; Eunice Kennedy Shriver National Institute of Child Health and Human Development Pelvic Floor Disorders Network. Refractory urgency urinary incontinence treatment in women: Impact of age on outcomes and complications. Am J Obstet Gynecol. 2017;218(1):111.e1-111.e9.

42. Cohen BL, Caruso DJ, Kanagarajah P, Gousse AE. Predictors of response to intradetrusor botulinum toxin-A injections in patients with idiopathic overactive bladder. Adv Urol. 2009:328364.

43. Liao $\mathrm{CH}$, Kuo HC. Increased risk of large post-void residual urine and decreased long-term success rate after intravesical onabotulinumtoxinA injection for refractory idiopathic detrusor overactivity. J Urol. 2013;189(5):1804-1810.

44. Hsiao SM, Lin HH, Kuo HC. Factors associated with therapeutic efficacy of intravesical onabotulinumtoxinA injection for overactive bladder syndrome. PLoS One. 2016;11(1):e0147137.

45. Makovey I, Davis T, Guralnick ML, O'Connor RC. Botulinum toxin outcomes for idiopathic overactive bladder stratified by indication: Lack of anticholinergic efficacy versus intolerability. Neurourol Urodyn. 2011;30(8):1538-1540.

46. Sievert KD, Chapple C, Herschorn S, et al. OnabotulinumtoxinA $100 \mathrm{U}$ provides significant improvements in overactive bladder symptoms in patients with urinary incontinence regardless of the number of anticholinergic therapies used or reason for inadequate management of overactive bladder. Int J Clin Pract. 2014;68(10):1246-1256.

47. Chohan N, Hilton P, Brown K, Dixon L. Efficacy and duration of response to botulinum neurotoxin $\mathrm{A}$ (onabotulinumA) as a treatment for detrusor overactivity in women. Int Urogynecol J. 2015;26(11):1605-1612.

48. Tincello DG, Owen RK, Slack MC, Mayne C, Toozs-Hobson P, Abrams KR. Efficacy of repeat treatment with onabotulinum toxin for refractory detrusor overactivity: Secondary analysis of open label extension of a randomized trial. Int Urogynecol J. 2014;25(Suppl 1):S1-S240.

49. Marcelissen TA, Rahnama'i MS, Snijkers A, Schurch B, De Vries P. Long-term follow-up of intravesical botulinum toxin-A injections in women with idiopathic overactive bladder symptoms. World $J$ Urol. 2017;35(2):307-311.

50. Denys $P$, Dmochowski R, Aliotta $P$, et al. Positive outcomes with first onabotulinumtoxinA treatment persist in the long term with repeat treatments in patients with neurogenic detrusor overactivity. BJU Int. 2017;119(6):926-932.

51. Owen RK, Abrams KR, Mayne C, Slack M, Tincello DG. Patient factors associated with onabotulinum toxin A treatment outcome in women with detrusor overactivity. Neurourol Urodyn. 2017;36(2):426-431.

52. Kessler TM, Danuser H, Schumacher M, Studer UE, Burkhard FC. Botulinum A toxin injections into the detrusor: An effective treatment in idiopathic and neurogenic detrusor overactivity? Neurourol Urodyn. 2005;24(3):231-236.

53. Davis NF, Burke JP, Redmond EJ, Elamin S, Brady CM, Flood HD. Trigonal versus extratrigonal botulinum toxin-A: A systematic review and meta-analysis of efficacy and adverse events. Int Urogynecol J. 2015;26(3):313-319.

54. Khan S, Kessler TM, Apostolidis A, et al. What a patient with refractory idiopathic detrusor overactivity should know about botulinum neurotoxin type a injection. J Urol. 2009;181(4):1773-1778. 
55. Collins L, Sathiananthamoorthy S, Fader M, Malone-Lee J. Intermittent catheterization after botulinum toxin injections: The time to reassess our practice. Int Urogynecol J. 2017;28(9):1351-1356.

56. Nitti VW, Ginsberg D, Sievert KD, et al; 191622-096 Investigators. Durable efficacy and safety of long-term onabotulinumtoxinA treatment in patients with overactive bladder syndrome: Final results of a 3.5-year study. J Urol. 2016;196(3):791-800.

57. Osborn DJ, Kaufman MR, Mock S, Guan MJ, Dmochowski RR, Reynolds WS. Urinary retention rates after intravesical onabotulinumtoxinA injection for idiopathic overactive bladder in clinical practice and predictors of this outcome. Neurourol Urodyn. 2015;34(7):675-678.

58. Jiang $\mathrm{YH}, \mathrm{Ong} \mathrm{HL}$, Kuo HC. Predictive factors of adverse events after intravesical suburothelial onabotulinumtoxina injections for overactive bladder syndrome-A real-life practice of 290 cases in a single center. Neurourol Urodyn. 2017;36(1):142-147.

59. Sahai A, Sangster P, Kalsi V, Khan MS, Fowler CJ, Dasgupta P. Assessment of urodynamic and detrusor contractility variables in patients with overactive bladder syndrome treated with botulinum toxin-A: Is incomplete bladder emptying predictable? BJU Int. 2009;103(5):630-634

60. Miotla P, Cartwright R, Skorupska K, et al. Urinary retention in female $\mathrm{OAB}$ after intravesical Botox injection: Who is really at risk? Int Urogynecol J. 2017;28(6):845-850.

61. Wang CC, Liao CH, Kuo HC. Diabetes mellitus does not affect the efficacy and safety of intravesical onabotulinumtoxinA injection in patients with refractory detrusor overactivity. Neurourol Urodyn. 2014;33(8):1235-1239.

62. Rodriguez LV, Blander DS, Dorey F, Raz S, Zimmern P. Discrepancy in patient and physician perception of patient's quality of life related to urinary symptoms. Urology. 2003;62(1):49-53.

63. Hikita KS, Honda M, Hirano S, et al. Comparison of the overactive bladder symptom score and the overactive bladder symptom score derived from the bladder diaries. Neurourol Urodyn. 2016;35(3):349-353.

64. Khan S, Panicker J, Roosen A, et al. Complete continence after botulinum neurotoxin type $A$ injections for refractory idiopathic detrusor overactivity incontinence: Patient-reported outcome at 4 weeks. Eur Urol. 2010;57(5):891-896.

65. Colman S, Chapple C, Nitti V, Haag-Molkenteller C, Hastedt C, Massow U. Validation of treatment benefit scale for assessing subjective outcomes in treatment of overactive bladder. Urology. 2008;72(4):803-807.
66. Hermieu JF, Ballanger P, Amarenco G, et al. Guidelines for practical usage of botulinum toxin type A (BoNTA) for refractory idiopathic overactive bladder management [in French]. Prog Urol. 2013;23(17):1457-1463.

67. Rovner E. Chapter 6: Practical aspects of administration of onabotulinumtoxinA. Neurourol Urodyn. 2014;33 (Suppl 3):S32-37.

68. Kaviani A, Khavari R. Disease-specific outcomes of botulinum toxin injections for neurogenic detrusor overactivity. Urol Clin North Am. 2017;44(3):463-474.

69. Jimenez-Cidre MA, Arlandis-Guzman S; en representacion del Grupo Espanol para el uso de Toxina Botulinica en U. OnabotulinumtoxinA in overactive bladder: Evidence-based consensus recommendations. Actas Urol Esp. 2016;40(3):139-147.

70. Kuo HC. Preliminary results of suburothelial injection of botulinum a toxin in the treatment of chronic interstitial cystitis. Urol Int. 2005;75(2):170-174.

71. Giannantoni A, Costantini E, Di Stasi SM, Tascini MC, Bini V, Porena M. Botulinum $A$ toxin intravesical injections in the treatment of painful bladder syndrome: A pilot study. Eur Urol. 2006;49(4):704-709.

72. Kennelly M, Dmochowski R, Ethans K, et al. Long-term efficacy and safety of onabotulinumtoxinA in patients with urinary incontinence due to neurogenic detrusor overactivity: An interim analysis. Urology. 2013;81(3):491-497.

73. Comperat E, Reitz A, Delcourt A, Capron F, Denys P, Chartier-Kastler E. Histologic features in the urinary bladder wall affected from neurogenic overactivity: A comparison of inflammation, edema and fibrosis with and without injection of botulinum toxin type A. Eur Urol. 2006;50(5):1058-1064.

74. Bottet F, Peyronnet B, Boissier R, et al; Groupe d'Etude de Neuro-Urologie de Langue Française (GENULF) and the committee of NeuroUrology of the French Association of Urology (AFU). Switch to abobotulinum toxin A may be useful in the treatment of neurogenic detrusor overactivity when intradetrusor injections of onabotulinum toxin A failed. Neurourol Urodyn. 2017;37(1):291-297.

75. Weckx F, Tutolo M, De Ridder D, van der Aa F. The role of botulinum toxin $A$ in treating neurogenic bladder. Transl Androl Urol. 2016;5(1):63-71. 